\title{
Opto-Electronic Investigation of Rubidium Based Fluoro-Perovskite for Low Birefringent Lens Materials
}

\author{
Iqbal, M. $\mathrm{A}^{1}$. and Erum, N. ${ }^{2}$ ** \\ ${ }^{1}$ Department of Physics, University of Management and Technology, Lahore \\ ${ }^{2}$ Department of Physics, University of Punjab, Lahore \\ *erum.n@hotmail.com
}

\begin{abstract}
In this communication, systematic first principles calculation has been scrutinize to evaluate bonding nature, structural, electronic, and optical properties of $\mathrm{RbHgF}_{3}$. The findings are based on total energy calculations where Khon Sham $(K S)$ equation is solved by means of density functional theory (FP-LAPW) method. Optimization of structural parameters is done with variety of approximations, which corroborates through comparison with available experimental data. Assessment of band profile through GGA plus Trans-Blaha modified Becke-Johnson (TB-mBJ) potential highlights underestimation of bandgap with traditional Generalized Gradient approximations. Specific contribution of particular states on electronic properties is investigated by means of total and partial density of states while contour maps of electron density are used to sightsee bonding character and it is evaluated that emphasized compound is $(M-\Gamma)$ indirect bandgap material with mixed ionic and covalent bonding character. Additionally attention is paid to absorption and reflection spectra of RbHgF $F_{3}$ luoroperovskite by reconnoitering optical properties, which shows extensive absorption and reflection in high frequency regions. Expectantly, current study would benchmark various quantum mechanical effects, which must be taken into account to understand and utilize $\mathrm{RbHgF}_{3}$ in fabricating practical devices.
\end{abstract}

Keywords: first- principles study, fluorine based perovskites, electronic property, optical property

\section{Introduction}

The knowledge of physical properties of materials is always a principal field of curiosity but in today's hi-tech era trust of potential investors can be strengthened by engrossment of researchers in competition towards higher proficiencies. To fulfill this need material scientists \& researchers struggling hard for economical and resourceful materials. The class of compounds having $\mathrm{ABF}_{3}$ stoichiometry is known as fluoroperovskites. Here A is usually alkali, alkaline or rare earth metals while $\mathrm{B}$ is supposed to be transition, post transition and non-transition metals although anion is represented by $\mathrm{X}$ that are oxides and halides [1]. 
In this study emphasize is given to Rubidium based mercury fluoroperovskite which own great technological importance in fabricating low birefringent excellent lens materials, transparent coating devices, opto-electronic \& optical pathways, photovoltaic applications, ionic conducting, luminescence capacitor, UV detectors as well as Light Emitting Diodes (LED). Particularly $\mathrm{RbHgF}_{3}$ is a superior choice for the high-class lens materials because it does not suffer with birefringence, which can make design of lenses problematic [2-10]. Comprehensive experimental studies are available on their interesting structural properties which is being confirmed that under consideration compound crystallizes in cubic structure and it do not unveil any phase transition under varies pressure and temperature [11-12].

From above literature this idea can be established that focused material is technically sound which motivate us to inquire it in detail. Our main goal is to improve trends of all physical properties concurrently. This paper is schematized in the following sections where Section 1, have already described some introductory details about materials and their applications. Section 2, is devoted to method and computational detail of calculations. Section 3, present results, and discussions of structural and opto-electronic properties. Finally, all results are compared with previous studies where data is available. At the end, we enlighten future prospective of this research.

\section{Computational Details}

The present first principles study have been carried out on the basis of density functional theory (DFT) which is implemented in wien2k code to solve Kohn Sham equation within Full Potential Linearized Augmented Plane Wave (FP-LAPW) method. This method is one of the best methods for appropriate computation of electronic states and optical response of various crystalline solids. For structural optimization the exchange correlation approximation is treated with Perdew-Burke-Ernzerhof Generalized Gradient Approximation (PBE-GGA), Wu Cohen Generalized Gradient Approximation (WC-GGA), PBEsol-GGA and Local Density Approximation (LDA) while for opto-electronic properties recently bugged GGA plus Trans-Blaha modified Becke-Johnson (TB-mBJ) potential is employed [13-16].

In order to enhance accuracy in calculations valence electrons are treated semi-relativistically and core electrons are treated fully relativistically. Convergence in basis size is achieved with a cut-off $\mathrm{R}_{\mathrm{MT}} \cdot \mathrm{K}_{\max }=8.0$, which is product of smallest muffin-tin radius RMT times the largest plane wave vector $\mathrm{K}_{\max }$. Brillouin zone (BZ) integration is done with $56 \mathrm{~K}$ point using modified form of tetrahedron method [17]. The calculations are self-consistently converged when total energy and charge is stable within $0.001 \mathrm{mRy}$ and $0.01 \mathrm{~m}$ respectively.

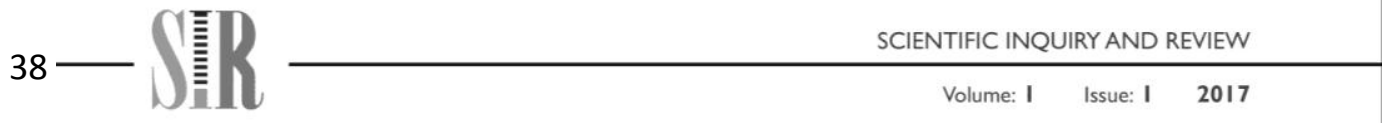


Furthermore, to calculate validate optical properties denser mesh of Brillouin zone (BZ) sampling is done with enormous number of $\mathrm{K}$ points.

Table1.

Comparison of Present calculation with previous experimental and theoretical values for lattice constants $\left(\mathrm{a}_{\mathrm{o}}\right)$, ground state energies $\left(\mathrm{E}_{\mathrm{o}}\right)$, bulk modulus $\left(\mathrm{B}_{\mathrm{o}}\right)$ and its pressure derivative $\left(\mathrm{B}_{\mathrm{p}}\right)$ of $\mathrm{RbHgF}_{3}$ compound.

\begin{tabular}{|c|c|c|c|c|c|c|}
\hline \multirow[t]{2}{*}{$\begin{array}{l}\text { Compound } \\
\mathrm{RbHgF}_{3}\end{array}$} & \multirow{2}{*}{$\frac{\text { Present work }}{\text { PBE-GGA }}$} & \multirow{2}{*}{$\frac{\text { Present work }}{\text { WC-GGA }}$} & \multirow{2}{*}{$\begin{array}{c}\text { Present work } \\
\text { - } \\
\text { PBEsol- } \\
\text { GGA }\end{array}$} & \multirow{2}{*}{$\frac{\begin{array}{c}\text { Present } \\
\text { work }\end{array}}{\text { LDA }}$} & \multirow{2}{*}{$\begin{array}{c}\text { Experi } \\
\text { mental } \\
\text { work }\end{array}$} & \multirow{2}{*}{$\begin{array}{c}\text { Other } \\
\text { theoretic } \\
\text { al work }\end{array}$} \\
\hline & & & & & & \\
\hline $\mathbf{a}_{\mathbf{0}}(\AA)$ & 4.60 & 4.57 & 4.53 & 4.49 & $4.47^{\mathrm{a}}$ & $4.46^{\mathrm{b}}$ \\
\hline $\mathbf{E}_{\mathbf{o}}(\mathbf{R y})$ & -45854.51 & -45854.43 & -45854.40 & -45854.39 & & \\
\hline $\mathbf{B}_{0}$ (GPa) & 48.84 & 49.01 & 49.39 & 49.81 & $48.32^{\mathrm{a}}$ & \\
\hline $\mathbf{B}_{\mathbf{P}}(\mathbf{G P a})$ & 5.61 & 5.58 & 5.53 & 5.51 & & \\
\hline
\end{tabular}

a) Ref.[22] (Experimental work)

b) Ref.[23] (Other theoretical work)

\section{Results and Discussion}

\subsection{Structural Properties}

Ternary fluoroperovskite $\mathrm{RbHgF}_{3}$ crystallizes itself in cubic type of structure having space group Pm3m (\#221) while its unit cell occupy one molecule. Figure1 illustrates the sites of Wyckoff coordinates which are situated at 1a $\left(\begin{array}{lll}0 & 0 & 0\end{array}\right), 1 \mathrm{~b}(1 / 2,1 / 2,0), 3 \mathrm{c}(0,1 / 2,1 / 2)$ for $\mathrm{Rb}, \mathrm{Hg}$ and $\mathrm{F}$ respectively. This subsection is dedicated to calculate structural properties of $\mathrm{RbHgF} 3$ via energy minimization process in which total energy varies as a function of equilibrium cell volume $\left(\mathrm{V}_{\mathrm{o}}\right)$. Accurate total energy versus volume curve is fitted with equation of state (EOS) developed by Murnaghan [18] as shown in Figure2.

Equilibrium lattice constant $\left(\mathrm{a}_{\mathrm{o}}\right)$, ground stata energy $\left(\mathrm{E}_{\mathrm{o}}\right)$, bulk modulus $\left(\mathrm{B}_{\mathrm{o}}\right)$, and pressure derivative of bulk modulus $\left(\mathrm{B}_{\mathrm{P}}\right)$ are calculated with four different exchange and correlation schemes namely PBE-GGA, WC-GGA, LDA and PBEsol-GGA. However from Table1 it can be observed that lattice constant computed by DFT slightly overvalue as compared to experimental data that can be associated due to use of traditional DFT schemes. The value of bulk modulus represents good crystal rigidity in $\mathrm{RbHgF}_{3}$ fluoroperovskite compound. Furthermore, Table1 depicts contrary relation between lattice constant and bulk modulus in accordance with the trend of other fluoroperovskite compounds [1921]. As a result all structural parameters are in reasonable agreement with previous theoretical and existing experimental data. 


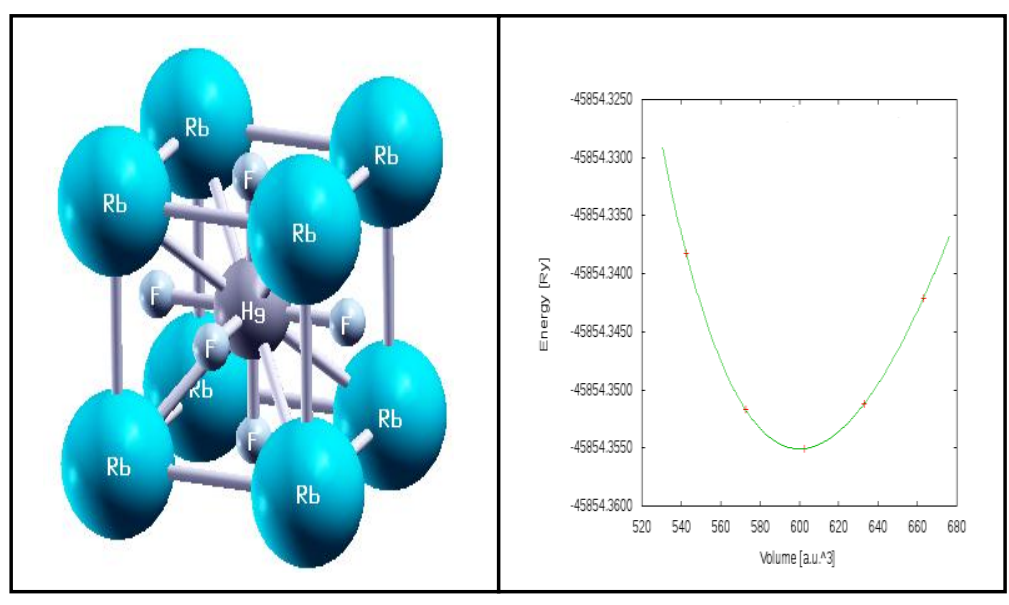

Figure1. Crystal structures of $\mathrm{RbHgF}_{3}$
Figure2. Variation of total energy as a function of unit cell volume for $\mathrm{RbHgF}_{3}$

\subsection{Electronic Properties}

The electronic properties of $\mathrm{RbHgF}_{3}$ fluoroperovskite compound is described in terms of calculating energy band structure and total as well as partial density of states (TDOS and PDOS) whereas bonding nature is evaluated with aid of electron density plots. In this subsection band gap is computed with GGA plus Trans-Blaha modified Becke-Johnson (TB-mBJ) potential in order to avoid underestimation of well-known exchange and correlation scheme of LDA and GGA [24-27]. To clarify this concept bandstructure comparison is made with PBE-GGA scheme to prove this underestimation. The resultant energy bandstructure in high symmetry directions are shown in Figure3 with PBE-GGA and $\mathrm{mBJ}$ potential. It can be analyzed from s that overall trend of band dispersion curves are almost same and conduction band minimum $(\mathrm{CBM})$ lies at $\Gamma$ symmetry point of brillouin zone (BZ) whereas valence band maximum (VBM) is located at $\mathrm{M}$ point brillouin zone (BZ) which reveals $(\mathrm{M}-\Gamma)$ indirect bandgap of $3.2 \mathrm{eV}$ and $1.0 \mathrm{eV}$ from $\mathrm{mBJ}$ and PBE-GGA schemes respectively. Unfortunately, there is lack of experimental bandgap data to make a reasonable comparison. However, the compound can work well in ultraviolet region of electromagnetic spectrum because the materials with band gaps larger than $3.1 \mathrm{eV}$ work well in the ultraviolet region of the spectrum [28].

Energy density distribution of varied states is observed in terms of total and partial density of states (TDOS \& PDOS). According to Figure4-5 it is obvious that TDOS and PDOS of $\mathrm{RbHgF}_{3}$ fluoroperovskite compound can be splitted into varied energy regions ranging from $-10 \mathrm{eV}$ to $15 \mathrm{eV}$. A narrow sharp peak is observed at $-10 \mathrm{eV}$ due to $\mathbf{R b}-\mathbf{4 p}$ states. The upper region of valence band

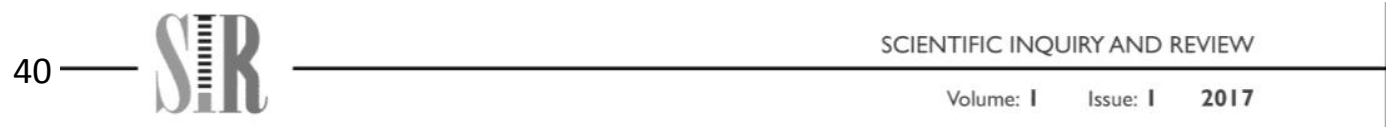


from Fermi-level to $-6.1 \mathrm{eV}$ is due to overlapping of $\mathbf{H g - 3 d}$ and $\mathbf{F - 2 p}$ states. While above Fermi level, lower part of conduction band is occupied by $\mathbf{H g}-\mathbf{4 s}$ and upper part of conduction band is filled by $\mathbf{R b}-\mathbf{4 d}$ states. For the sake of precision in density of states, we omit some distorted peaks which can hinder justification of electronic states.
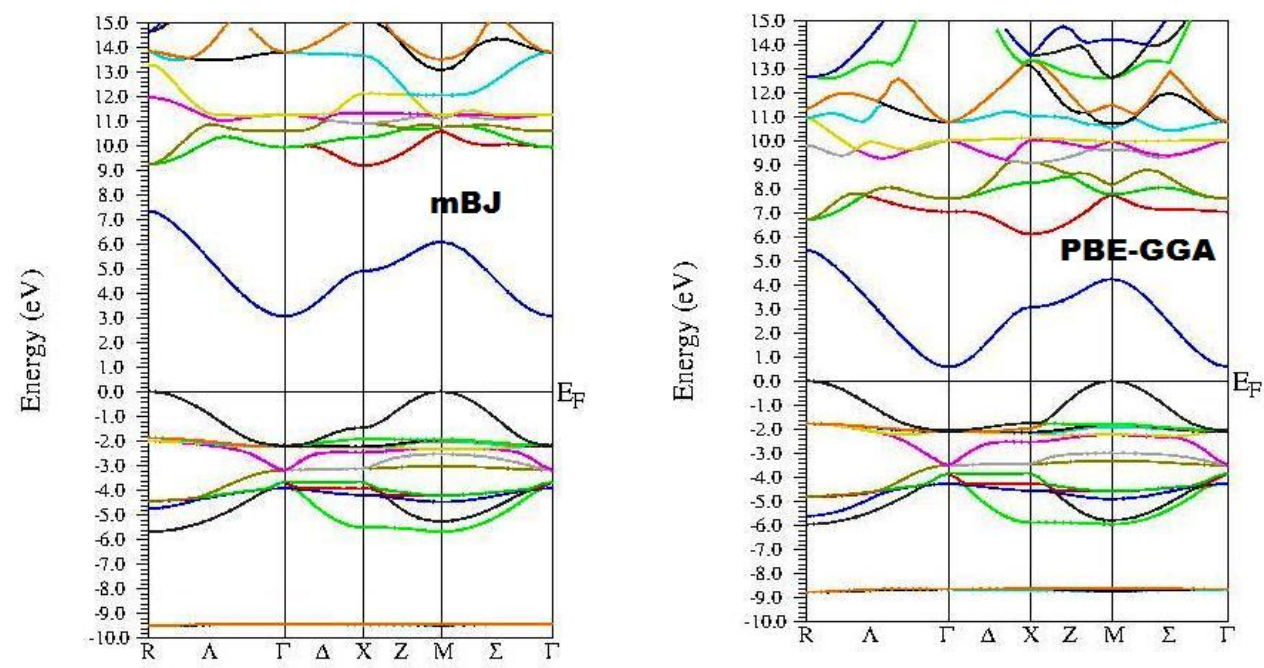

Figure3. Comparison of calculated band structure in high symmetry directions with PBEGGA \& mBj potential
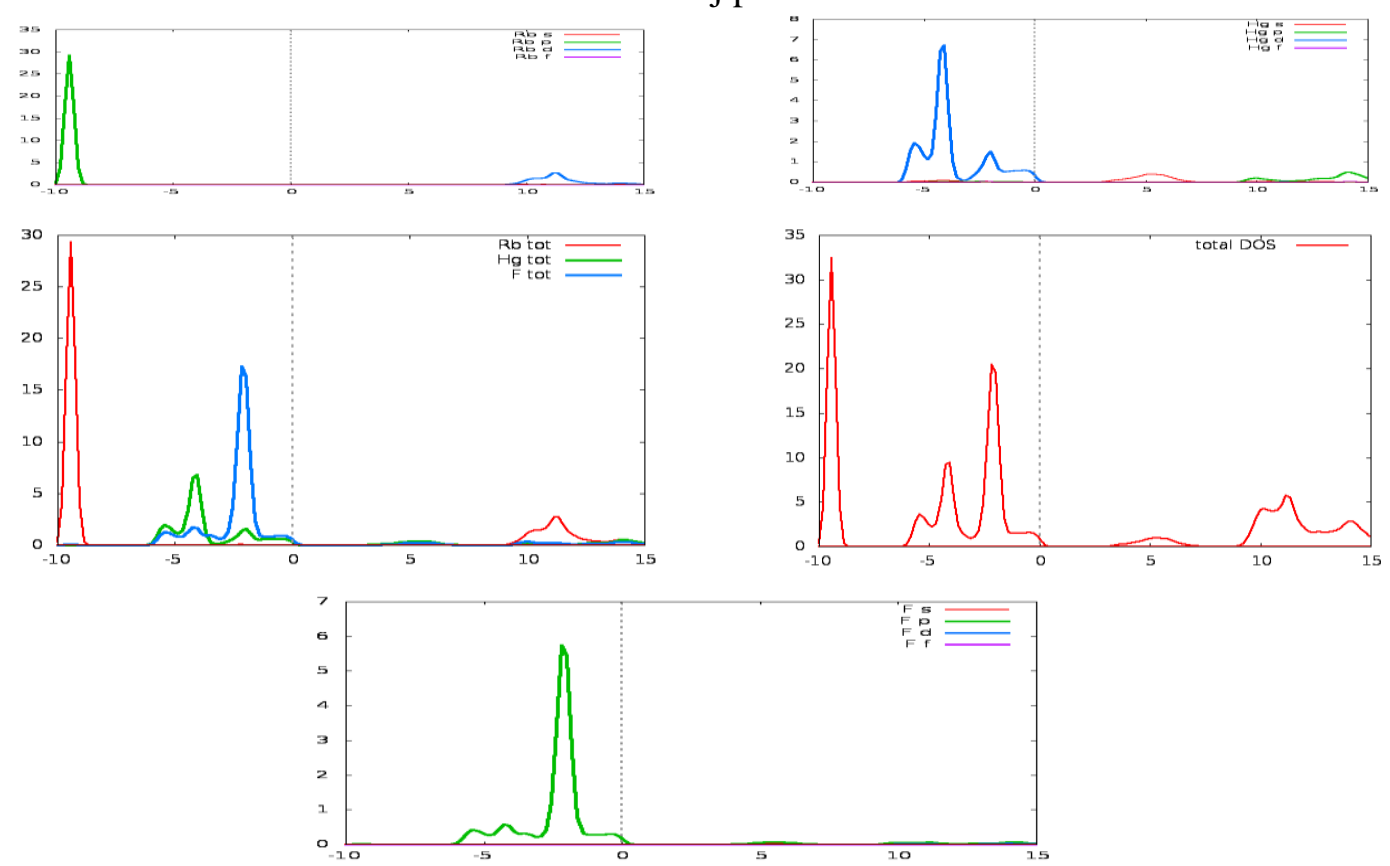

Figure4-5 Calculated total and partial density of states (TDOS \& PDOS)

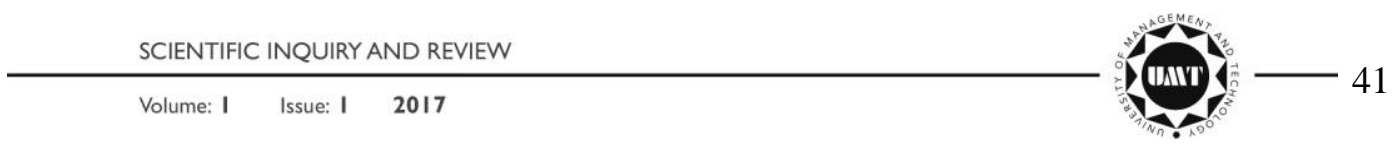



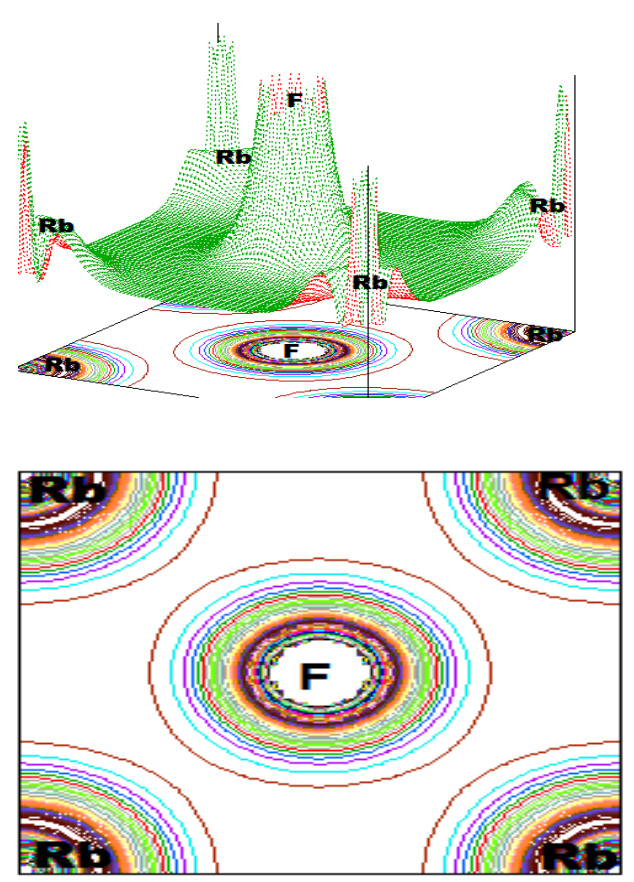

(I) (100) plane
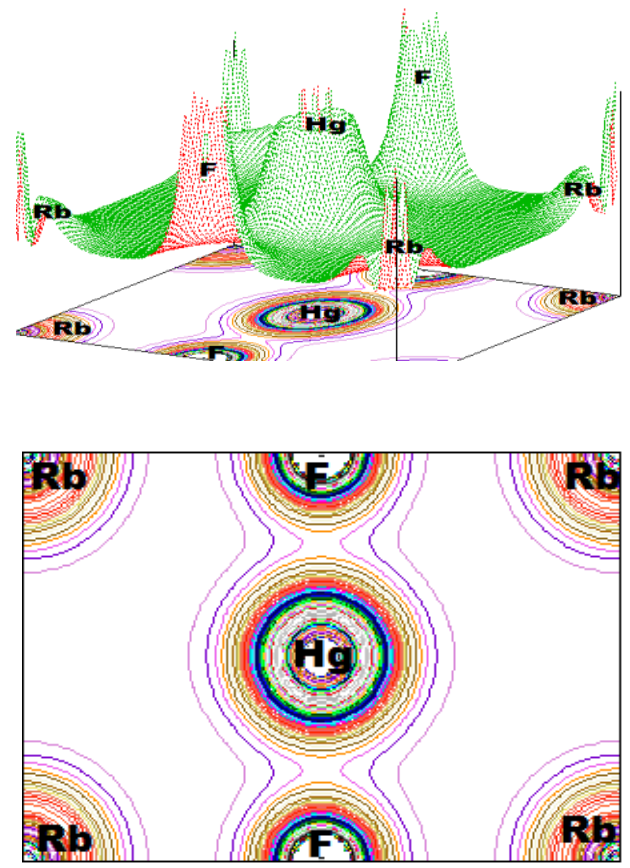

(II) (110) plane

Figure6. Calculated $\mathrm{mBJ}$ total two and three dimensional electronic charge densities for (I) in the (100) plane and (II) in the (110) plane.

The maps of contour plots in terms of electron density communicate a crucial role in explaining nature of chemical bond in crystalline materials [29]. The dispersion curves of contour maps are calculated along (100) and (110) planes in $2 \mathrm{D}$ alongwith $3 \mathrm{D}$ as quoted in Figure6. The strong ionic nature of $\mathrm{Rb}-\mathrm{F}$ bond is evident from perfect spherical charge distribution between cation of rubidium and anion of fluorine. The charge is transferred between Rbcation and $\mathrm{F}$ anion due to large electronegativity difference in them. On the other hand, uniform distribution is observed between $\mathrm{Hg}$ cation and $\mathrm{F}$ anion which divulges covalent nature in $\mathrm{HgF}_{3}$ octahedra. In fact above analysis synchronizes well with results of DOS Figure4-5 where p-d hybridization is maximum between $\mathbf{H g - 3 d}$ and F-2p states. Conclusively our results authorize mixed ionic as well as covalent bonding nature in $\mathrm{RbHgF}_{3}$. Furthermore in Refs. [20,21] similar results of bonding type is observed for other fluoroperovskite compounds.

In order to expose internal behavior of emphasized compound the tool of optical analysis is employed. Fundamental optical responses include imaginary part of dielectric function $\varepsilon_{2}(\omega)$, real part dielectric function $\varepsilon_{1}(\omega)$. Further analysis is extended to plot optical spectra of absorption coefficient and reflectivity which are crucial in concern with device applications. 
The complex dielectric function $\varepsilon(\omega)$ can break into real and imaginary part according to following relation [30]:

$$
\varepsilon(\omega)=\varepsilon_{1}(\omega)+i \varepsilon_{2}(\omega)
$$

In equation (1) $\varepsilon_{2}(\omega)$ denotes imaginary part of dielectric function while $\varepsilon_{1}(\omega)$ represent real part of dielectric function. The analysis of $\varepsilon_{2}(\omega)$ as shown in Figure7 out complete response of material due to applied electromagnetic radiation. In this study, we highlight results of direct interband transition although summarizing all possible transitions originating from occupied valence band to unoccupied conduction band taking into account appropriate element of transition dipole matrix [31].The widespread peaks of $\varepsilon_{2}(\omega)$ follow pattern of DOS and band structure of the investigated compound. The threshold energy point occurs at $5.2 \mathrm{eV}$ approximately while the major peak are located at $5.9 \mathrm{eV}$ which corresponds to transition of occupied valence band states to unoccupied conduction band states. These peaks are majorly due to $\mathrm{Hg}-4 \mathrm{~s}$ states. After that diverse peaks are observed till $15 \mathrm{eV}$ which occurs due to hybridized states of $\mathrm{Rb}$ $4 \mathrm{~d}$ alongwith some $\mathrm{p}$ states of $\mathrm{Hg}$ as well as $\mathrm{F}$.

Absorptive behavior of material is analyzed with the help of real part of dielectric function $\varepsilon_{1}(\omega)$ as shown in Figure8. At zero frequency limit static part of dielectric function $\varepsilon_{1}(0)$ is observed at $1.88 \mathrm{eV}$. The curves of $\varepsilon_{1}(\omega)$ starts increasing and attains a maximum value at around $5 \mathrm{eV}$ and an overall narrow bandgap semiconductive nature is observed.

The plot of absorption coefficient as a function of energy depicts that $\mathrm{RbHgF} 3$ starts absorbing electromagnetic radiation at about $5.25 \mathrm{eV}$ as presented in Figure9. This particular energy is also known as threshold point. This threshold point is exactly in accordance with trend of bandgap. Hereafter oscillations are observed with increasing absorption pattern in spectra. Investigated material starts absorbing effectively within $21-25 \mathrm{eV}$ range while highest prominent peak is observed at around $21.5 \mathrm{eV}$. After the incent of optimum absorption peak it again going to decrease suffering trivial variations. Analysis of absorption spectra concludes clearly about application of this fluoroperovskite for absorption purposes in wide range of Ultra-Violet region of electromagnetic spectrum typically at about $21.5 \mathrm{eV}$.

The calculated spectrum of reflectivity as a function of electromagnetic energy is shown in Figure10. The phenomenon of reflectivity stays below 9-10\% upto $20 \mathrm{eV}$. Currently focused material starts reflecting highly and attains maximum value almost at $22 \mathrm{eV}$ that helps to understand that by following different regions of electromagnetic spectrum (EM) $\mathrm{RbHgF}_{3}$ remains highly transparent in infrared (IR) and visible (V) regions which recommends that under

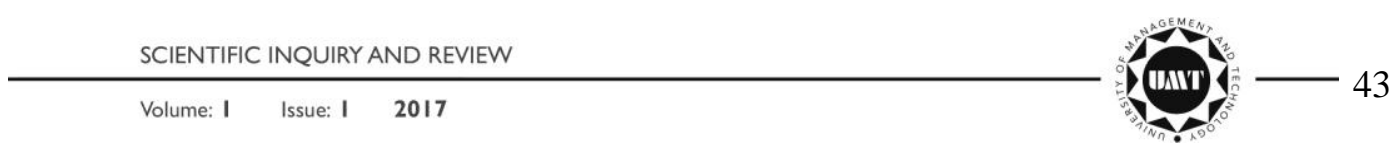


consideration compound is an upbeat candidate for efficient lenses and transparent coating devices.

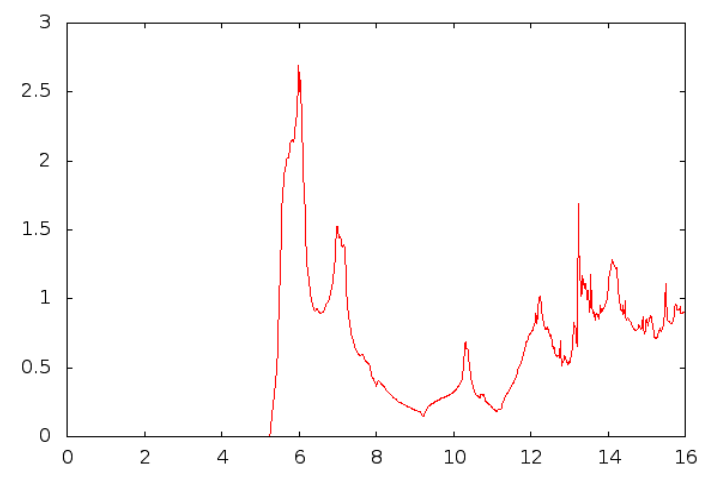

Figure7. Calculated imaginary part $\varepsilon_{2}(\omega)$ of the di-electric function for $\mathrm{RbhgF}_{3}$ compound
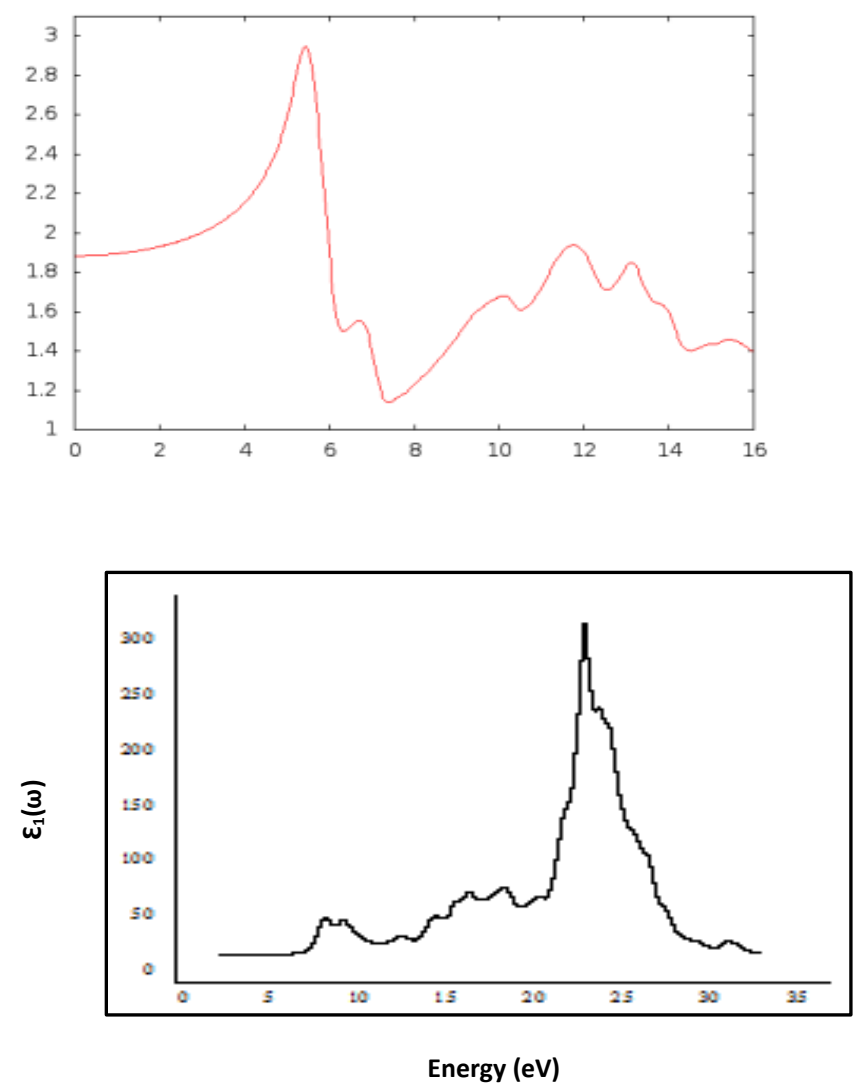

Figure8. Calculated real part $\varepsilon_{1}(\omega)$ of the dielectric function for $\mathrm{RbHgF}_{3}$ compound. 


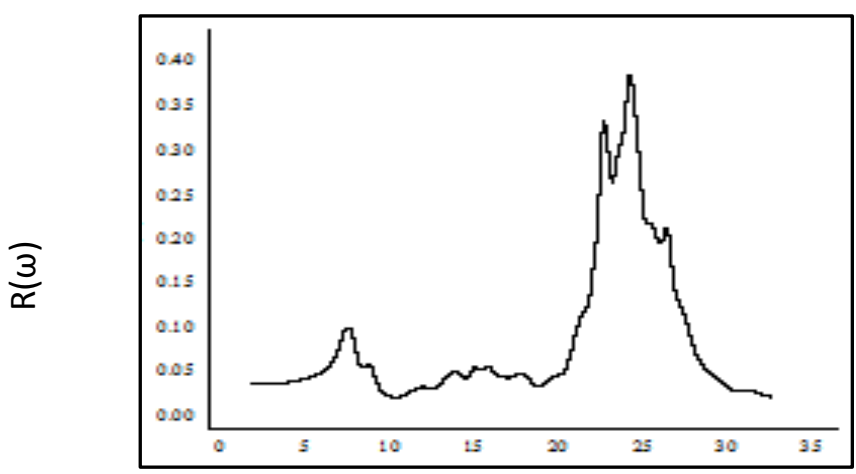

Energy (eV)

Figure9. Reflectivity $\mathrm{R}(\omega)$ as a function of energy for $\mathrm{RbHgF}_{3}$ compound

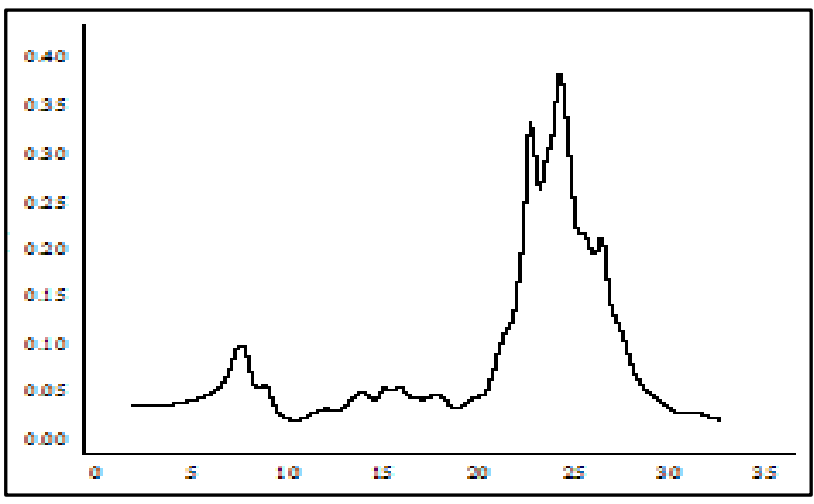

Figure10. Absorption coefficient $\alpha(\omega)$ as a function of energy for $\mathrm{RbHgF}_{3}$ compound

\section{Conclusion}

In this work, all electron self-consistent Full Potential-Linearized Augmented Plane Wave (FP-LAPW) method is used to explore structural, electronic, and optical properties of $\mathrm{RbHgF}_{3}$ fluoro-perovskite compound. In order to inspect optimized structural parameters exchange-correlation contribution is treated with four different approximations. To elude underestimation of band profile by other DFT schemes we highlight GGA plus Trans-Blaha modified Becke-Johnson (TB-mBJ) potential in lieu of attaining opto-electronic results near to expected experimental findings. Energy band profile confirms that investigated material is a narrow and indirect energy bandgap $(\mathrm{M}-\Gamma)$ semicondutor. The total and partial density of state curves is used to define 
contribution of different bands. In addition to it contour maps of electron density authenticates mixed covalent-ionic behavior. These results are in favorable agreement with previous theoretical and existing experimental data. The optical properties are discussed in terms of advantageous functional parameters and analysis is being done by interband contribution that shows $\mathrm{RbHgF}_{3}$ possess wide range of absorption and reflection in high frequency regions and these characteristics make them useful for transparent coatings and fabricating low birefringent high-quality lenses. Conclusively limelight fluoroperovskitecan efficiently be used for fabricating high-class low birefringentlense material and photovoltaic applications.

\section{References}

[1] Lang L, Yang J, Liu H, Xiang HJ, Gong XG. First-principles study on the electronic and optical properties of cubic $\mathrm{ABX}_{3}$ halide perovskites. Phy Lett A. 2014;378(3):290-293.

[2] Vaitheeswaran G, Kanchana V, Svane A, Delin A. High-pressure structural study of fluoroperovskite $\mathrm{CsCdF} 3$ up to $60 \mathrm{GPa}$ : A combined experimental and theoretical study. J Phys: Condens Matter. 2007;19:326214.

[3] Eibschutz M, Guggenheim HJ, Wemple SH, Camlibel I, Didomenico M. Ferroelectricity in BaM+2 F4. Phys Lett. 1969;7:409.

[4] Horsch G, Paus HJ. A new color center laser on the basis of lead doped $\mathrm{KMgF}_{3}$. Opt Commun. 1986;60:69.

[5] Salehi H. First Principles studies on the electronic structure and band structure of paraelectric SrTiO3 by different approximations. J Mod Phys. 2011;2:934-943.

[6] Slassi A. Ab initio study of a cubic perovskite: Structural, electronic, optical andelectrical properties of native, lanthanum and antimony-doped barium tin oxide. Mater Sci Semicond Process. 2015;32:100-106.

[7] Chadwick AV, Strange JH. Studies of ionic motion in perovskite fluorides. In: Gesellschaft DP, editor. Physikalische Berichte, vol 6. Berlin: Physik Verlag; 1983. p. 9-10, 555-558.

[8] Vaills Y, Buzare JY. X-ray investigations of the cubic to tetragonal phase transition in $\mathrm{CsCaCl} 3$ at Tc $=95$ K. Solid State Commun. 1986;60:139-141.

[9] Mitchell RH. Perovskites: Modern and ancient. Thunder Bay, Ont., Canada: Almaz Press; 2002.

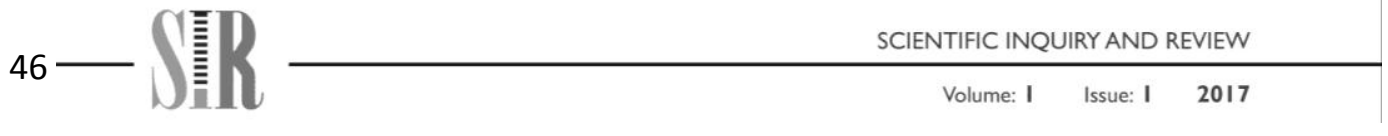


[10] Huang K, Feng M, Goodenough J, Milliken C. Double perovskites as anode materials for solid oxide fuel cells. J Electrochem Soc. 1997;144:3620.

[11] Ihringer J, Maichle J, Prandl W, Hewat A, Wroblewski T. Crystal structure of the ceramic superconductor $\mathrm{BaPb}_{0.75} \mathrm{Bi}_{0.25} \mathrm{O}_{3}$. Zeitschrift Fur Physik BCondensed Matter. 1991;82:171.

[12] Dotzler C, Williams GV, Edgar A. Radiation-induced optically and thermally stimulated luminescence in $\mathrm{RbCdF} 3$ and $\mathrm{RbMgF} 3$. Curr Appl Phys. 2008;8:447.

[13] Schwarz K, Blaha P. Solid state calculations using Wien2k. Comput Mat Sci. 2003;28:259-273.

[14] Blaha P, Schwarz K, Madsen GK. Electronic structure calculations of solids using the WIEN2k package for material sciences. Comput Phys Commun. 2002; 147:71-76.

[15] Kohn W, Sham LJ. Self-consistent equations including exchange and correlation effects. Phys Rev. 1965;140:1133.

[16] Tran F, Blaha P. Accurate band gaps of semiconductors and insulators with a semilocal exchange-correlation potential. Phys Rev Lett. 2009;102:226401.

[17] Khetre SM, Jadhav HV, Jagadale PN, Kulali SR, Bamane SR. Studies on electrical and dielectric properties of LaFeO3. Adv Appl Sci Res. 2011;2:503511.

[18] Murnaghan FD. The compressibility of media under extreme. Pressures Proc Natl Acad Sci USA. 1994;3:244.

[19] Ghebouli B, Ghebouli MA, Bouhemadou A, Fatmi M, Khenata R, Rached D, et al. First principles study of the structural, elastic, electronic, optical and thermodynamic properties of the cubic perovskite $\mathrm{CsCdCl} 3$ under high pressure. Solid State Sci. 2012;14:903.

[20] Rose SK, Satpathy S, Jepsen O. Semiconducting CsSnBr3. Phys Rev B. 1993;47:4276-4280.

[21] Brik MG. Comparative first-principles calculations of electronic, optical and elastic anisotropy properties of $\mathrm{CsXBr} 3(\mathrm{X}=\mathrm{Ca}, \mathrm{Ge}, \mathrm{Sn})$ crystals. Solid State Commun. 2011;151:733-1738.

[22] Muller O, Roy R. The major ternary structural families. New York: Springer; 1974. 
[23] Moreira RL, Dias A. Comment on prediction of lattice constant in cubic perovskites. J Phys Chem Solids. 2007;68:1617.

[24] Berastegui P, Hull S. Eriksson SG. A low-temperature structural phase transition in CsPbF3. J Phys: Condens Matter. 2001;13(22):5077.

[25] Reshak AH, Chen X, Auluck S, Kamarudin H. DFT calculation for elastic constants of orthorhombic structure within WIEN2K code: A new package (ortho-elastic). J Appl Phys. 2012;112:053526.

[26] El Haj Hassan F, Akbarzadeh H. First-principles elastic and bonding properties of barium chalcogenides. Comput Mater Sci. 2006;38:362-368.

[27] Reshak AH, Kamarudin H, Auluck S. DFT calculation for elastic constants of orthorhombic structure within WIEN2K code: A new package (orthoelastic). J Phys Chem B. 2012;116:4677.

[28] Luana V, Costales A, Pendas AM, Florez M, Fernandez VM. Structural and chemical stability of halide perovskites. Solid State Commun. 1997;104:4750 .

[29] Kittel C, Introduction to solid state physics. 8th ed. New York: John Wiley \& Sons; 2005.

[30] Fox, optical properties of solids. New York: Oxford University Press; 2001.

[31] Kumar S, Maury TK, Auluck S. Correlation between ionic charge and the lattice constant of cubic perovskite solids. J Phys: Condens Matter. 2008;20:075205.

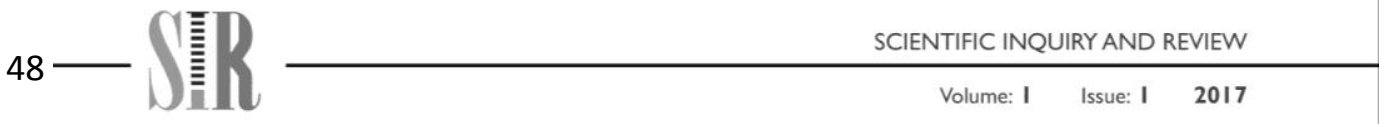

